\title{
Symmetry breaking and spin lattice coupling in $\mathrm{NdCrTiO}_{5}$
}

\author{
K. Gautam, ${ }^{1}$ A. Ahad, ${ }^{2}$ K. Dey, ${ }^{1}$ S. S. Majid, ${ }^{2}$ S. Francoual, ${ }^{3}$ V. G. Sathe, ${ }^{1}$ Ivan da Silva, ${ }^{4}$ and D. K. Shukla ${ }^{1, *}$ \\ ${ }^{1}$ UGC-DAE Consortium for Scientific Research, Khandwa Road, Indore 452001, India \\ ${ }^{2}$ Department of Physics, Aligarh Muslim University, Aligarh 202002, India \\ ${ }^{3}$ Deutsches Elektronen-Synchrotron, Notkestrasse 85, D-22607 Hamburg, Germany \\ ${ }^{4}$ ISIS Facility, Rutherford Appleton Laboratory, Chilton, Didcot OX11 0QX, United Kingdom
}

(Received 6 March 2019; revised manuscript received 2 July 2019; published 9 September 2019)

\begin{abstract}
The origin of multiferroicity in $\mathrm{NdCrTiO}_{5}$ has been examined. A first-order phase transition, due to breaking of the inversion symmetry, near the low-temperature antiferromagnetic ordering $\left(T_{N} \sim 21 \mathrm{~K}\right)$ where spontaneous electric polarization also appears, is confirmed. From synchrotron x-ray powder diffraction measurements, the structure at low temperatures is found to be noncentrosymmetric (space group Pba2), justifying the polar behavior of this compound below $T_{N}$. Temperature-dependent Raman measurements reveal that the lattice is strongly coupled with the spins, pointing to the spin lattice correlations in a noncentrosymmetric space group being responsible for invoking the ferroelectricity below $21 \mathrm{~K}$. Through time-of-flight neutron powder diffraction experiments, it is confirmed that the magnetic sublattices, both $\mathrm{Nd}$ and $\mathrm{Cr}$, simultaneously order at $T_{N}$, and at temperatures below $15 \mathrm{~K}$ the $\mathrm{Cr}$ moments are found to be saturated while the Nd moments continue to grow until $6 \mathrm{~K}$.
\end{abstract}

DOI: 10.1103/PhysRevB.100.104106

\section{INTRODUCTION}

The coexistence of magnetic and ferroelectric orders in multiferroic materials introduces the possibility of controlling magnetization by the electric field and vice versa [1], making those compounds extremely interesting for technological applications [2,3]. In these type of materials, spontaneous ferroelectricity emerges due to the magnetic ordering. On the other hand, magnetoelectric (ME) materials exhibit strong magnetic-field-dependent anomalies in the electrical polarization and the dielectric measurements. Compounds with the formula $A B_{2} \mathrm{O}_{5}$ (where $A$ denotes a rare-earth ion and $B$ is a transition-metal ion) exhibit both of the above characteristics $[4,5]$. In these compounds, the crystal structure is generally very well coupled with emerging magnetic ordering and electric polarization. Strong coupling between the magnetic order and the lattice was recently observed in $\mathrm{TbMn}_{2} \mathrm{O}_{5}$ [6]. Wang et al. [7] showed that the ferroelectricity in this compound is driven by the magnetic ordering, which breaks the inversion symmetry.

A member of this family, $\mathrm{NdCrTiO}_{5}$, has attracted attention due to its strong magnetoelectric (ME) coupling [8]. Roomtemperature crystal structure of $\mathrm{NdCrTiO}_{5}$ belongs to the orthorhombic Pbam space group [8,9], with four chemical formulas per unit cell, which is a centrosymmetric space group, from the point group $\mathrm{mmm}$. Hwang et al. showed that $\mathrm{NdCrTiO}_{5}$ orders antiferromagnetically at $T_{N} \sim 21 \mathrm{~K}$, and they described the possibilities of the multiferroicity and the ME effect, both due to observations of the ME annealing effect as well as spontaneous electric polarization [8]. Recently, Saha et al. [9] established that $\mathrm{NdCrTiO}_{5}$ is a genuine multiferroic by showing a nondecaying pyroelectric

*dkshukla@csr.res.in current in absence of a magnetic field over time. The driving mechanism of the multiferroicity is not very clear. It is either due to the Dzyaloshinskii-Moriya (DM) interaction [10], which is generally responsible for spontaneous polarization due to interaction between the noncollinear spins, or due to the magnetostriction, which explains the ME effect through the spin lattice coupling when spins order collinearly [11]. Recently, with the help of a systematic substitution study in $\mathrm{NdCrTiO}_{5}$, Kori et al. [12] highlighted the cooperative interaction between the $4 f$ and the $3 d$ magnetic moments over the $3 d$ magnetic moment interactions, and the noncollinear arrangement of the $\mathrm{Nd}$ and the $\mathrm{Cr}$ moments near the magnetic transition temperature. However, it shows spontaneous polarization with a centrosymmetric crystal structure, which is not very clear. An answer to the above questions can come from high-resolution structural measurements at low temperatures, across the magnetic transition, but these studies are scarce. In this paper, we report on detailed low-temperature structural studies using synchrotron $\mathrm{x}$-ray powder diffraction and Raman spectroscopy on multiferroic $\mathrm{NdCrTiO}_{5}$. Our results unravel a noncentrosymmetric space group and a strong spin lattice coupling at low temperatures, presenting a rationale behind genuine multiferroicity in $\mathrm{NdCrTiO}_{5}$ below $T_{N}$.

\section{EXPERIMENTAL DETAILS}

Polycrystalline $\mathrm{NdCrTiO}_{5}$ was prepared through the conventional solid-state reaction method. High-purity powders of the oxides $\mathrm{Nd}_{2} \mathrm{O}_{3}(99.9) \%, \mathrm{Cr}_{2} \mathrm{O}_{3}(99.9) \%$, and $\mathrm{TiO}_{2}(99.9) \%$ were thoroughly mixed to achieve stoichiometry and then sintered at $1200{ }^{\circ} \mathrm{C}$ for $24 \mathrm{~h}$ with intermediate grindings. $\mathrm{X}$-ray diffraction experiments were performed at beamline P09 at PETRA-III at DESY (Hamburg, Germany) in transmission mode at $23 \mathrm{keV}(\lambda=0.5386 \AA)$ [13]. A large-area Perkin Elmer XRD1621 detector with a $40 \times 40 \mathrm{~cm}^{2}$ active 

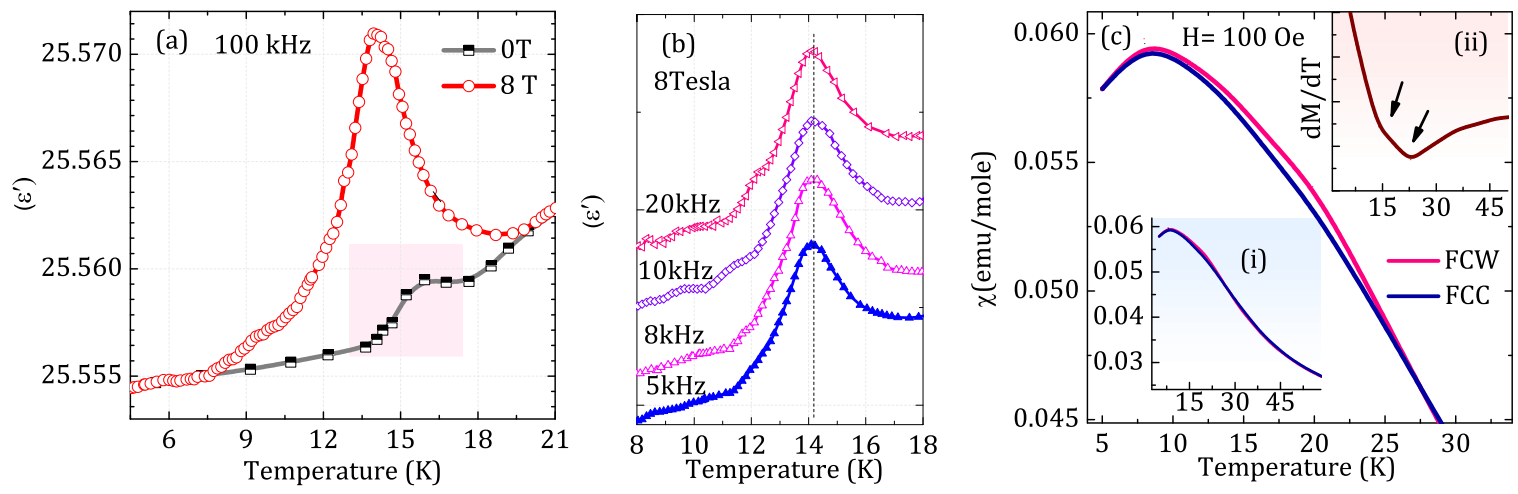

FIG. 1. (a) Temperature-dependent dielectric permittivity in the presence of 0 and $8 \mathrm{~T}$ magnetic field. (b) Temperature dependence of the dielectric permittivity peaks (at $8 \mathrm{~T}$ ) at various frequencies. (c) Magnetization vs temperature curves under the FCC and the FCW protocols, measured in the presence of 100 Oe magnetic field. Inset (i) shows an overview of the FCC and the FCW curves. (ii) The $d M / d T$ vs temperature.

area, $2048 \times 2048$ pixels, and $200 \mu \mathrm{m}^{2}$ pixel size was used for data collection. Magnetization measurements as a function of temperature and magnetic field were performed by employing a commercial 7-T SQUID vibrating sample magnetometer (SVSM; Quantum Design Inc., USA) in zero-field-cooled (ZFC), field-cooled (FC), and field-cooled-warming (FCW) cycles with an applied field of 100 Oe. For dielectric measurements, the sample was prepared in a 10-mm-diameter disk shape, and silver electrodes were deposited on both surfaces. Dielectric measurements were performed utilizing a Hioki LCR meter IM3536 and a CRYOCON temperature controller, with an ac excitation voltage of $1 \mathrm{~V}$ inside a superconducting cryomagnet (OXFORD). Raman measurements were carried out using a LABRAM HR-800 spectrometer from Horiba (JY, Japan) equipped with a $488 \mathrm{~nm}$ excitation source, a 1800 grooves/mm grating, and a CCD detector. The position accuracy of the spectrometer is $\sim 0.33 \mathrm{~cm}^{-1}$ and the overall spectral resolution is $1 \mathrm{~cm}^{-1}$. Temperature-dependent timeof-flight (TOF) neutron powder diffraction (NPD) data were collected using the General Materials Diffractometer (GEM) [14] at the ISIS facility (UK).

\section{RESULTS}

\section{A. Dielectric and magnetization studies}

The main features in the low-temperature dielectric anomaly in $\mathrm{NdCrTiO}_{5}$ (shown in Fig. 1) are clearly associated with the magnetic transition temperature. The dielectric constants measured at 8 and $0 \mathrm{~T}$ show dielectric anomalies at $\sim 14$ and $\sim 16 \mathrm{~K}$, respectively [Fig. 1(a)]. Earlier, Hwang et al. [8] observed a systematic decrease of the transition temperature with increasing magnetic field. This is also observed here. Figure 1(b) shows the temperature dependence of the dielectric permittivity peak at $8 \mathrm{~T}$ at various frequencies. A frequency-independent behavior of the dielectric permittivity peak is indicative of classical-type ferroelectricity with a structural transition [15]. A similar behavior, but with a less pronounced peak feature, is also observed in zero magnetic field (not shown).

In magnetization measurements shown in Fig. 1(c), we observe an antiferromagnetic (AFM) ordering at $T_{N} \sim 21 \mathrm{~K}$, and a small, but clearly visible, hysteresis between the FCC and the FCW curves of the $M(T)$ indicates a first-order phase transition.

\section{B. Synchrotron $x$-ray powder diffraction}

The Rietveld refined pattern of room-temperature x-ray diffraction data of $\mathrm{NdCrTiO}_{5}$, using the reported Pbam space group, is presented in Fig. 2(a). The $\mathrm{NdCrTiO}_{5}$ polycrystalline
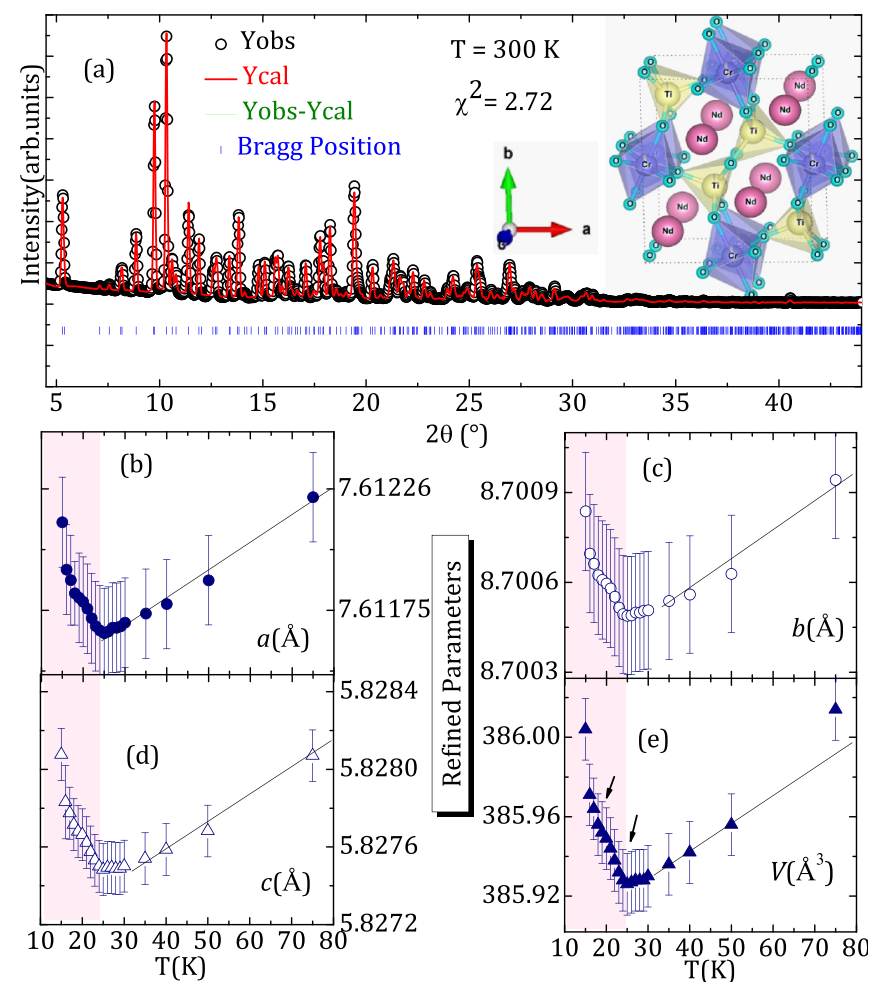

FIG. 2. (a) Synchrotron x-ray powder diffraction of the $\mathrm{NdCrTiO}_{5}$ at room temperature along with the Rietveld refinement. The inset shows a crystallographic arrangement of the ions in this compound, in the Pbam space group. (b)-(e) Temperature dependence of the refined lattice parameters and the unit-cell volume of the $\mathrm{NdCrTiO}_{5}$. 

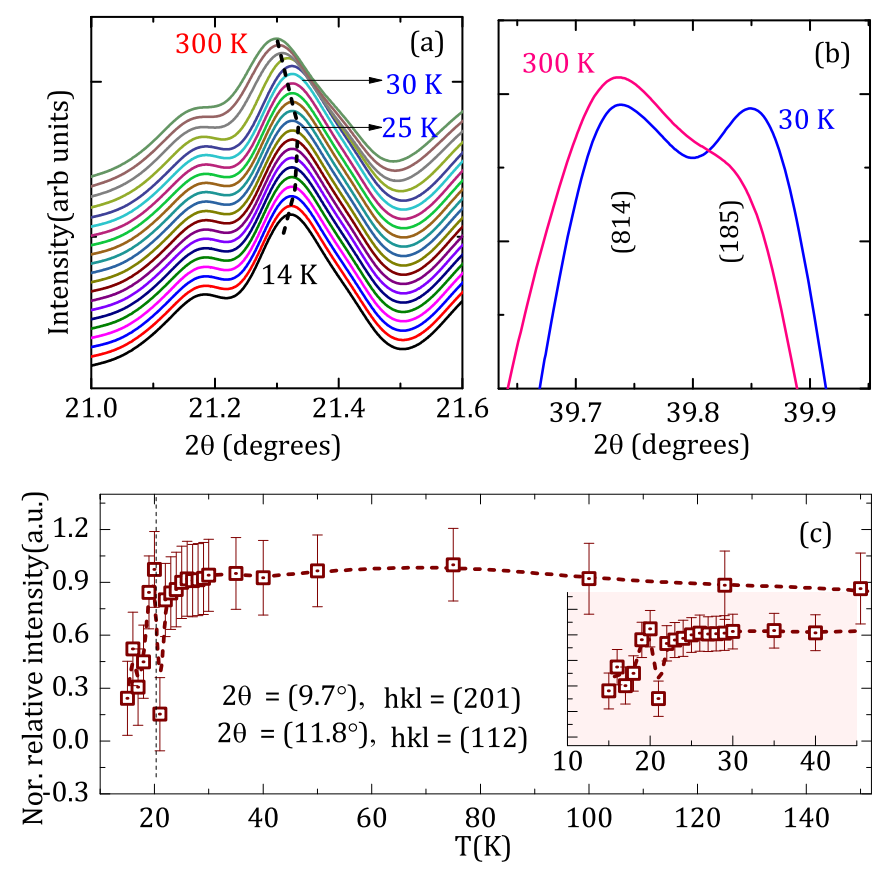

FIG. 3. (a) Zoomed view of a selected reflection (3 23 ), showing an unusual temperature behavior in the XRD pattern of $\mathrm{NdCrTiO}_{5}$. (b) Visuals of structural change evidenced through a change in intensity ratio of selected peaks at higher $2 \theta$ angles. (c) Temperature dependence of the ratio of the normalized integrated intensities of two selected reflections [( $\left.\begin{array}{lll}2 & 0 & 1\end{array}\right)$ and $\left(\begin{array}{lll}1 & 1 & 2\end{array}\right)$ ].

sample was found to be single-phase. The room-temperature crystal structure of $\mathrm{NdCrTiO}_{5}$ is orthorhombic, containing corner-sharing $\mathrm{Cr}^{+3} \mathrm{O}_{6}$ octahedra and the $\mathrm{Ti}^{+4}$ pyramids, while the $\mathrm{Nd}^{+3}$ ions are located at the interstitials [see the inset in Fig. 2(a)].

We have performed Rietveld refinement for the whole range of temperature-dependent $\mathrm{x}$-ray diffraction patterns of $\mathrm{NdCrTiO}_{5}$, with the centrosymmetric space group Pbam using the FULLPROF program [16]. The temperature dependence of the lattice parameters and the volume are shown in Figs. 2(b)-2(e). Anomalies in the lattice parameters coincide with anomalies in the $M(T)$ curve [shown in the derivative of the $M(T)$ curve; see inset (ii) of Fig. 1(c)], which indicate two transitions, one at $\sim 21 \mathrm{~K}$ and another at $\sim 15 \mathrm{~K}$. The variation of all the lattice parameters $a, b$, and $c$ obtained from fitting is demonstrating negative thermal expansion (NTE) type behavior below $\sim 25 \mathrm{~K}$ [see Figs. 2(b)-2(d)], which is unusual.

Thorough investigation shows an unusual temperature behavior of the XRD peaks below $\sim 30 \mathrm{~K}$. With a lowering of the temperature, the XRD peaks are moving toward lower $2 \theta$ angles [see Fig. 3(a)], and for some of the peaks the $2 \theta$ variations seem to be constant. The weaker reflections that are present at the higher $2 \theta$ angles are supposed to be most sensitive to structural changes, as these will offer better reciprocal space resolution $[17,18]$. Clear changes in the intensity ratios are observed at low temperatures compared to higher (room) temperature for higher-order reflections; see Fig. 3(b). We have also plotted a temperature variation of the ratio of the integrated diffraction intensities of Bragg reflections [(201)

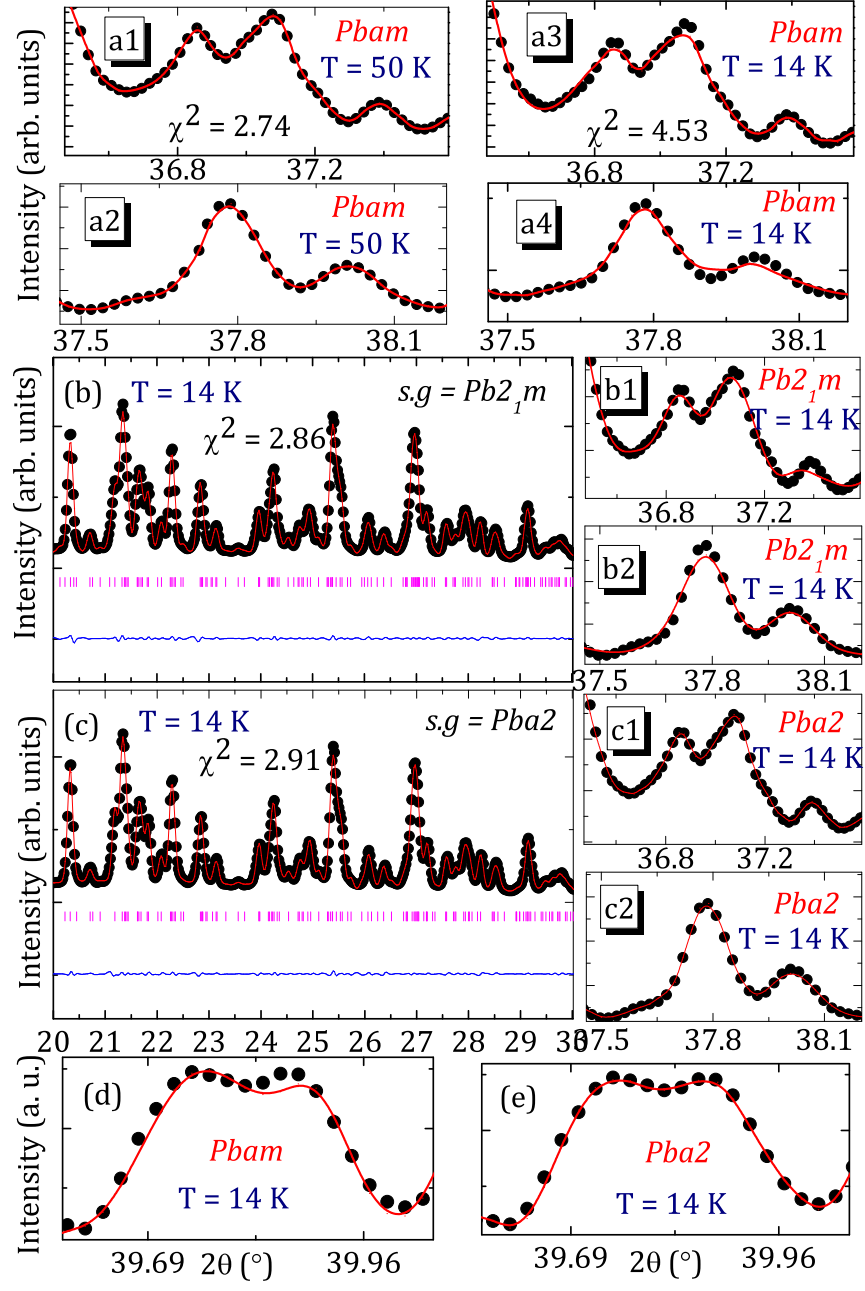

FIG. 4. The Rietveld refined x-ray diffraction patterns of $\mathrm{NdCrTiO}_{5}$ powder sample, using three different space groups: Pbam (a1-a4), $P b 2_{1} \mathrm{~m}(\mathrm{~b}-\mathrm{b} 2)$, and Pba2 (c-c2), along with the zoomed fittings at the higher $2 \theta$ values. Parts (d) and (e) show fittings of a higher angle reflection using Pbam and Pba2 space groups at $14 \mathrm{~K}$.

and (112)], shown in Fig. 3(c). The relative intensity of these reflections suddenly decreases below $\sim 20 \mathrm{~K}$, marking a clearcut structural transition around this temperature. Changes in the integrated diffraction intensities have been widely used as a tool to show a structural transition $[19,20]$.

The Pbam symmetry allows two relevant noncentrosymmetric space groups, $P b a 2$ and $P b 2_{1} m$, having the point group $m m 2$. These two space groups are the polar maximal subgroup of Pbam, and the group-subgroup index of 2 [ $G$ and $H$ represent group and subgroup $(G: H), H$ has index 2 in $G]$ [21].

Both of these groups are differentiated from each other with respect to the Pbam group in three aspects: (i) Pba2 is represented in the standard " $a b c$ " setting, while $P b 2_{1} m$ is in the " $b c a$ " setting. (ii) Reflection conditions for the Pba2 space group are exactly the same as the Pbam space group (i.e., $0 k l: k=2 n ; h 0 l: h=2 n ; h 00: h=2 n ; 0 k 0: k=2 n$ ), while reflection conditions for $P b 2_{1} m$ are different (i.e., $0 k$ $l: k=2 n ; 0 k 0: k=2 n)$. The space group $P b 2{ }_{1} m$ deals with splitting of the Wyckoff positions, $4 \mathrm{~g}$ into $2 \mathrm{a}$, and 2a for Nd, $4 \mathrm{f}$ into $2 \mathrm{~b}$, and $2 \mathrm{~b}$ for $\mathrm{Ti} / \mathrm{Cr}$, and the emergence of the additional 

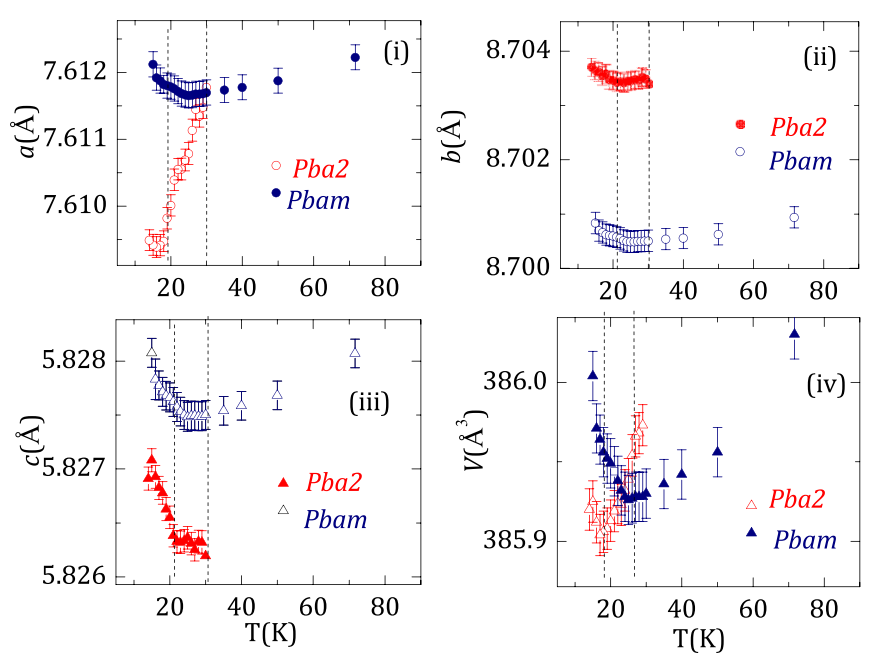

FIG. 5. Temperature dependence of the refined lattice parameters and the unit-cell volume of $\mathrm{NdCrTiO}_{5}$ using both space groups, Pbam (300-14 K) and Pba2 (below $30 \mathrm{~K}$ ).

reflections is expected in the x-ray diffraction data. (iii) In $P b a 2$, the $c$ axis serves as the polar axis whereas in the $P b 2_{1} m$ space group this is the $b$ axis. We have performed refinement of temperature-dependent x-ray diffraction data of $\mathrm{NdCrTiO}_{5}$ by considering all three space groups.

The x-ray diffraction data, when refined using the Pbam space group, shows an NTE anomaly (as discussed in the previous section), and we notice that at $14 \mathrm{~K}$ the peaks at the higher $2 \theta$ values are not well fitted [see Figs. 4(a3) and 4(a4)]. While considering refining the low-temperature $\mathrm{x}$-ray diffraction data using the polar space groups, $P b a 2$ and $P b 2_{1} m$, one has to remember the fact that $P b 2_{1} m$ should exhibit extra reflections, and, to our experimental resolution, we did not observe any extra reflection. However, based on the mere absence of the weak reflections, the possibility of the $P b 2_{1} m$ space group cannot be ignored. In the case of $\mathrm{TbMn}_{2} \mathrm{O}_{5}$, there was not enough visual evidence of the symmetry changes in the experimental data including the XRD [22]. Extremely small atomic displacements of the order of $10^{-4}$ were reported $[7,22]$. So we performed careful refinement for both space groups, which are found to fit low-temperature data better than Pbam; see Figs. 4(b) and 4(c). However, for the reflections at the higher $2 \theta$ values, fitting is best for the Pba2; see Fig. 4(c1,c2). Figures 4(d) and 4(e) show the refinements at $14 \mathrm{~K}$ using the Pbam as well as the Pba2 space group for the reflection shown in Fig. 3(b). The intensity profile of this higher angle reflection is fitted very well using the $P b a 2$ space group rather than Pbam. So, from our x-ray diffraction data we can conclude that the correct space group in the lowertemperature region below $T_{N}$ is the noncentrosymmetric space group Pba2.

We have combined the refinement results from both space groups Pbam (above $30 \mathrm{~K}$ ) and Pba2 (below $30 \mathrm{~K}$ ), shown in Figs. 5 and 6. Anisotropic temperature behavior in the lattice parameters is observed when refined with the Pba2 space group (see Fig. 5) as well as a negative thermal expansion, observed below $20 \mathrm{~K}$ in the refined unit-cell volume. In the $\mathrm{TiO}_{5}$ tetragonal pyramids, two different pairs among the four oxygens in the basal plane are defined as $\mathrm{O} 1$ and $\mathrm{O} 2$, and the apical oxygen is named $\mathrm{O} 3$. To describe the oxygens present in the $\mathrm{CrO}_{6}$ octahedron, the pair of basal oxygens are named $\mathrm{O}^{\prime}$ and $\mathrm{O} 2^{\prime}$, and the apical oxygen is named $\mathrm{O}^{\prime}$ ' [see the schematic diagram in Fig. 6(1)]. The temperature dependence of bond distances (Ti-O2, Ti-O3, Ti-O1, Cr-O1' ${ }^{\prime}, \mathrm{Cr}^{-} \mathrm{O} 2^{\prime}$, and $\mathrm{Cr}^{-} \mathrm{O3}^{\prime}$ ) are presented in Figs. 6(a)-6(f). We observe steplike
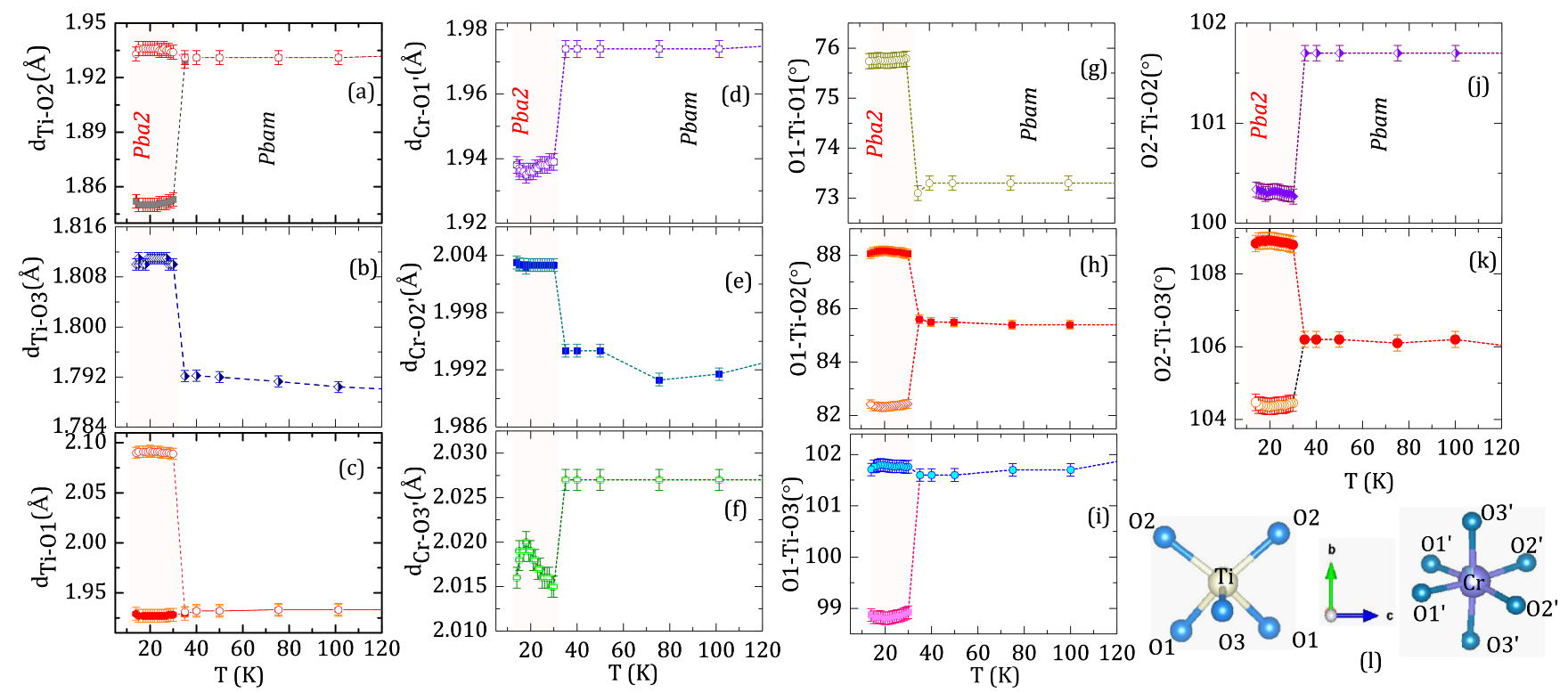

FIG. 6. Temperature dependence of the bond lengths and the angles of the atoms extracted through the Rietveld refinement in both space groups Pbam (300-30 K) and Pba2 (below $30 \mathrm{~K}$ ). (a)-(c) Bond lengths of Ti-O1, Ti-O2, and Ti-O3 in the TiO 5 structural unit. (d)-(f) Bond lengths of $\mathrm{Cr}_{-} \mathrm{O}^{\prime} / \mathrm{O}^{\prime} / \mathrm{O}^{\prime}$ in the $\mathrm{CrO}_{6}$ structural unit. $(\mathrm{g})-(\mathrm{k})$ Bond angles between the $\mathrm{Ti}$ and oxygens $\left(\mathrm{O} 1, \mathrm{O}_{2}\right.$, and $\left.\mathrm{O} 3\right)$ in $\mathrm{TiO} 5$ polyhedral. (1) Schematic of tetragonal pyramid and octahedron formed by the $\mathrm{TiO}_{5}$ and the $\mathrm{CrO}_{6}$ polyhedra, representing the position of oxygen present in apical and basal planes in the corresponding structural units, and the same are used in parts (a)-(k). 

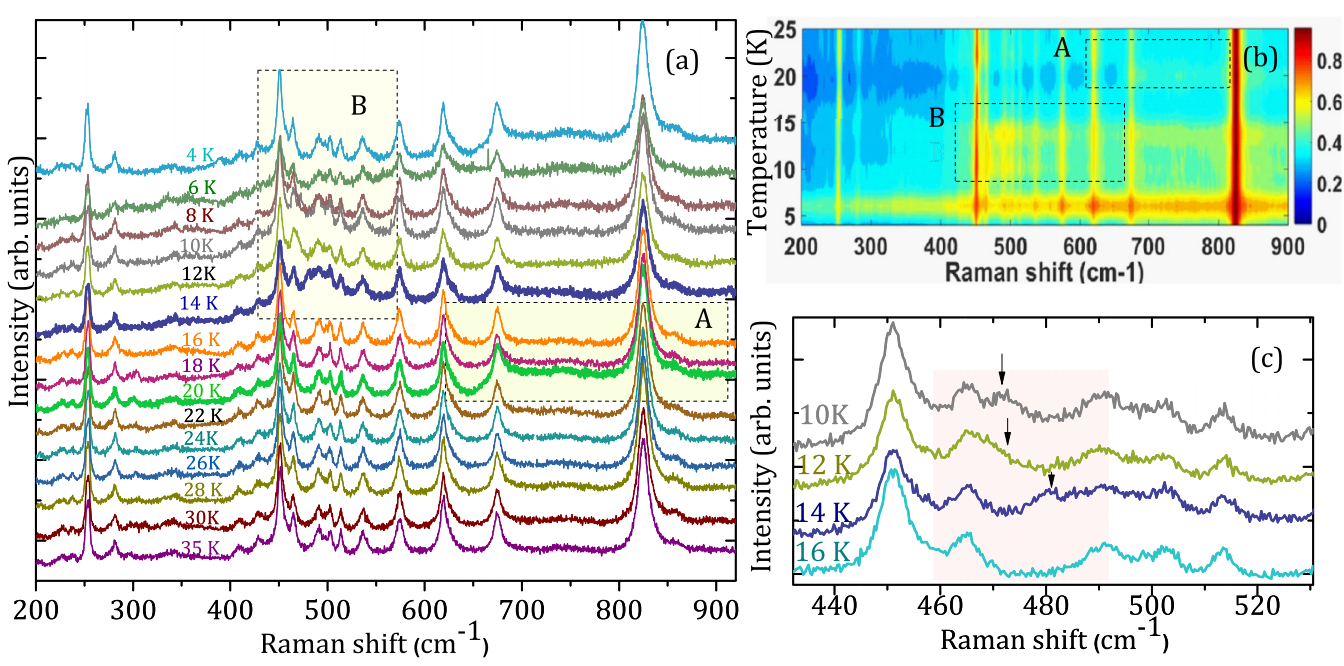

FIG. 7. (a) Temperature-dependent Raman spectra of $\mathrm{NdCrTiO}_{5}$. (b) Contour plot of the intensity vs the Raman shift for a clear vision of an increase in the background near magnetic transition temperatures. Rectangular boxes labeled $A$ and $B$ are used to highlight the temperature and frequency ranges where anomalous changes in the background intensity of the Raman spectra are observed. (c) Evolution of the new Raman modes in the low-temperature region.

changes near $30 \mathrm{~K}$ in all the Ti-O and $\mathrm{Cr}-\mathrm{O}$ bonds. The bond distances of $\mathrm{Ti}-\mathrm{O} 2$ and $\mathrm{Ti}-\mathrm{O} 1$ pairs are the same above $30 \mathrm{~K}$ (in the Pbam space group), and below this temperature the bond distances of the pairs Ti-O1 and Ti-O2 do not remain the same and get split into different bond distances. One of the bond distances in the Ti-O2 pair shows a smaller change (TiO2 $\sim 1.93 \AA$ ) while the other one shows a big steplike change (Ti-O2 $1.85 \AA$ ). Similar behavior is obtained for the Ti-O1 pair (Ti-O1 $\sim 2.09 \AA$ ), and a small change (Ti-O1 $\sim 1.94 \AA$ ) is observed [see Fig. 6(c)]. On the other hand, we do not observe any anisotropic distortion in the $\mathrm{CrO}_{6}$ octahedron. Only the magnitudes of the bond distances in both space groups are different, accompanied by a steplike transition. The NTE behavior below $20 \mathrm{~K}$ seems to be more connected to the bond distances of the Cr-O network. The distortion behavior is also accompanied by the angles between $\mathrm{O} 1-\mathrm{Ti}-\mathrm{O} 2$ and $\mathrm{O} 1-$ Ti-O3 and O2-Ti-O3 units [see Figs. 6(h), 6(i) and 6(k)]. All these results clearly indicate that the structural deformations are mainly realized in the $\mathrm{TiO}_{5}$ polyhedrons and the NTE behavior mostly in the $\mathrm{Cr}-\mathrm{O}_{6}$ octahedron.

\section{Raman scattering}

Raman scattering is a sensitive and powerful technique to see local structural changes across a phase transition. According to the symmetry analysis of the Pbam orthorhombic structure, there is a total of 96 modes,

$$
\begin{gathered}
\Gamma(\text { Pbam })=9 B_{1 u} \oplus 15 B_{2 u} \oplus 15 B_{3 u} \oplus 13 A_{g} \\
\oplus 13 B_{1 g} \oplus 11 B_{2 g} \oplus 11 B_{3 g} \oplus 9 A_{u},
\end{gathered}
$$

Raman-active modes $=48\left(13 A_{g}+13 B_{1 g}+11 B_{2 g}+11 B_{3 g}\right)$,

Infrared-active modes $=36\left(8 B_{1 u}+14 B_{2 u}+14 B_{3 u}\right)$,

Acoustical modes $=3\left(B_{1 u}+B_{2 u}+B_{3 u}\right)$,

silent modes $=9\left(A_{u}\right)$.
Figure 7 shows the temperature-dependent Raman spectra for $\mathrm{NdCrTiO}_{5}$. Not all the Raman-active modes are visible experimentally, probably due to low intensities and resolution limitation of the experimental setup. Based on the shell model calculation of the reference Raman spectra of the isostructural compounds $R \mathrm{Mn}_{2} \mathrm{O}_{5}(R=\mathrm{Ho}$,Dy) [23], we have divided our Raman spectra into the three frequency zones. (i) $100-350 \mathrm{~cm}^{-1}$; modes in between this frequency range generally correspond to vibration of heavier atoms like $\mathrm{Nd}$ and $\mathrm{Cr}$. The frequency region below $200 \mathrm{~cm}^{-1}$ most probably belongs to $\mathrm{Nd}$ atoms, and the range above is mostly dominated by the asymmetric stretching caused by the motion of the $\mathrm{Cr}$ atom in the $\mathrm{Cr}-\mathrm{O}-\mathrm{Cr}$ structural units. (ii) $350-550 \mathrm{~cm}^{-1}$; modes in this range belong to the asymmetric bending due to displacement of the $\mathrm{Cr} / \mathrm{Ti}$ atoms in the $\mathrm{Cr}-\mathrm{O}-\mathrm{Ti}$ structural units. (iii) $>550 \mathrm{~cm}^{-1}$; modes in this range are associated dominantly with the stretching mode of the $\mathrm{TiO}_{5}, \mathrm{Cr}-\mathrm{O}-\mathrm{Cr}$, and $\mathrm{Cr}-\mathrm{O}-\mathrm{Ti}$ structural units due to motion of the oxygen. Just by looking at our Raman data, the following direct observations can be made. First, in the frequency range above $\sim 650 \mathrm{~cm}^{-1}$, where the motion of the oxygen is supposed to be playing an important role, a sudden increase in the background is observed at around $20 \mathrm{~K}$ (see box A). Second, in the intermediate frequency range (ii), at low temperatures a broad hump (box B) suddenly appears that is maximum at $\sim 14 \mathrm{~K}$.We observe that the background spectral weight slowly transfers from the high-frequency to the low-frequency region near the magnetic transition temperature. This type of broad feature below $T_{N}$ attributed to magnetic ordering was also reported for an isostructural compound $\mathrm{GdCrTiO}_{5}$ [24,25]. Third, new Raman modes in the intermediate range (ii) are observed at low temperatures [see Fig.7(c)], indicative of structural distortion corresponding to $\mathrm{Cr}$ and Ti modes.

We have fitted the Raman modes by the Lorentzian fitting function and analyzed both the Raman shift and linewidth as a function of temperature. In Figs. 8(a), 8(c) and 8(e), one can notice that the phonon frequencies suddenly start to 

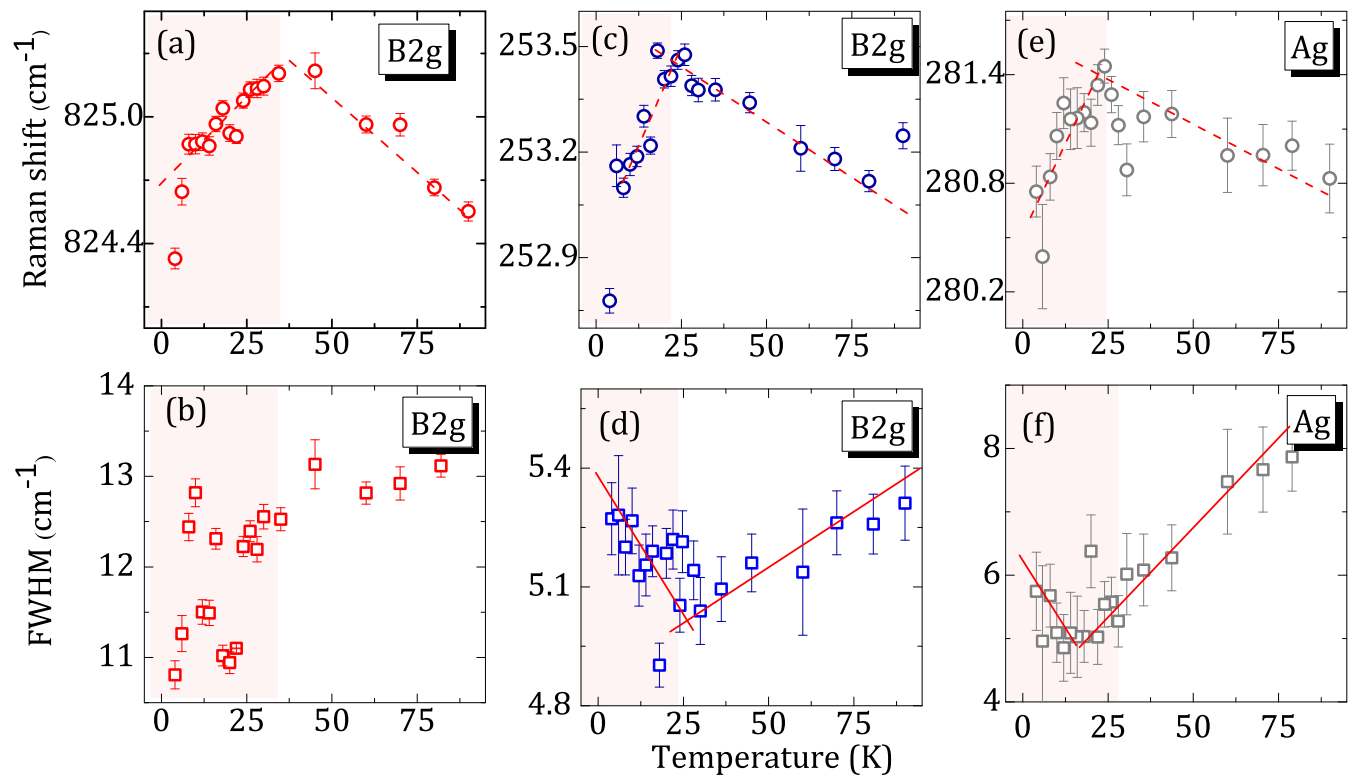

FIG. 8. (a), (c) and (e) Temperature dependence of the Raman shift positions of the $B_{2 g}\left(824 \mathrm{~cm}^{-1}\right), B_{2 g}\left(253 \mathrm{~cm}^{-1}\right)$, and $A_{g}\left(280 \mathrm{~cm}^{-1}\right)$ modes in $\mathrm{NdCrTiO}_{5}$. (b), (d) and (f) Linewidth temperature dependence of the modes $B_{2 g}\left(824 \mathrm{~cm}^{-1}\right), B_{2 g}\left(253 \mathrm{~cm}^{-1}\right)$, and $A_{g}\left(280 \mathrm{~cm}^{-1}\right)$ in $\mathrm{NdCrTiO}_{5}$.

decrease below the magnetic transition temperature, indicating softening of the modes and deviation from an intrinsic anharmonic contribution. Figure 8(a) shows a temperature dependence variation of the $B_{2 g}$ mode $\left(\sim 825 \mathrm{~cm}^{-1}\right)$, which is dominated by motion of the oxygen in the Ti-O-Ti structural unit. This mode becomes soft below $30 \mathrm{~K}$, and this transition temperature matches with the one obtained from the XRD data. The temperature evolution of the $B_{2 g}$ and $A_{g}$ modes ( $\sim 253$ and $\sim 281 \mathrm{~cm}^{-1}$ ), shown in Figs. 8(c) and 8(e), also indicate an anomalous behavior, softening of modes, but at around the AFM transition $(21 \mathrm{~K})$. Both of these modes belong to an asymmetric bending due to the displacement of the $\mathrm{Cr} / \mathrm{Ti}$ atom in the $\mathrm{Cr}-\mathrm{O}-\mathrm{Ti}$ structural unit. This type of transition from hardening to softening of phonon modes while going down in temperature is present in almost all the modes and indicates the presence of the strong spin lattice coupling at low temperatures. To further investigate more about the interaction between the spins and the lattice, it is crucial to analyze the behavior of the linewidth with temperature as the Raman linewidths are related to the phonon lifetime, which is unaffected by a lattice volume change but is considerably affected by the spin phonon coupling in insulating materials. Figures 8(b), 8(d) and 8(f) show the linewidth temperature dependence of the respective modes. Anomalies are visible in the modes with lower frequencies, which correspond to $\mathrm{Nd}$ and $\mathrm{Cr}$ sublattices, but no clear anomaly is observed for the higher-frequency mode. These results indicate a spin-phonon coupling dominated by $f$ - $d$ exchange interaction.

\section{Neutron powder diffraction}

Figures 9(a) and 9(b) show neutron powder diffraction patterns at $100 \mathrm{~K}$ (above the magnetic phase transition) for banks 3 and 5 covering higher and lower $d$-spacing ranges, respectively. Similarly, Figs. 9(c) and 9(d) show the refined
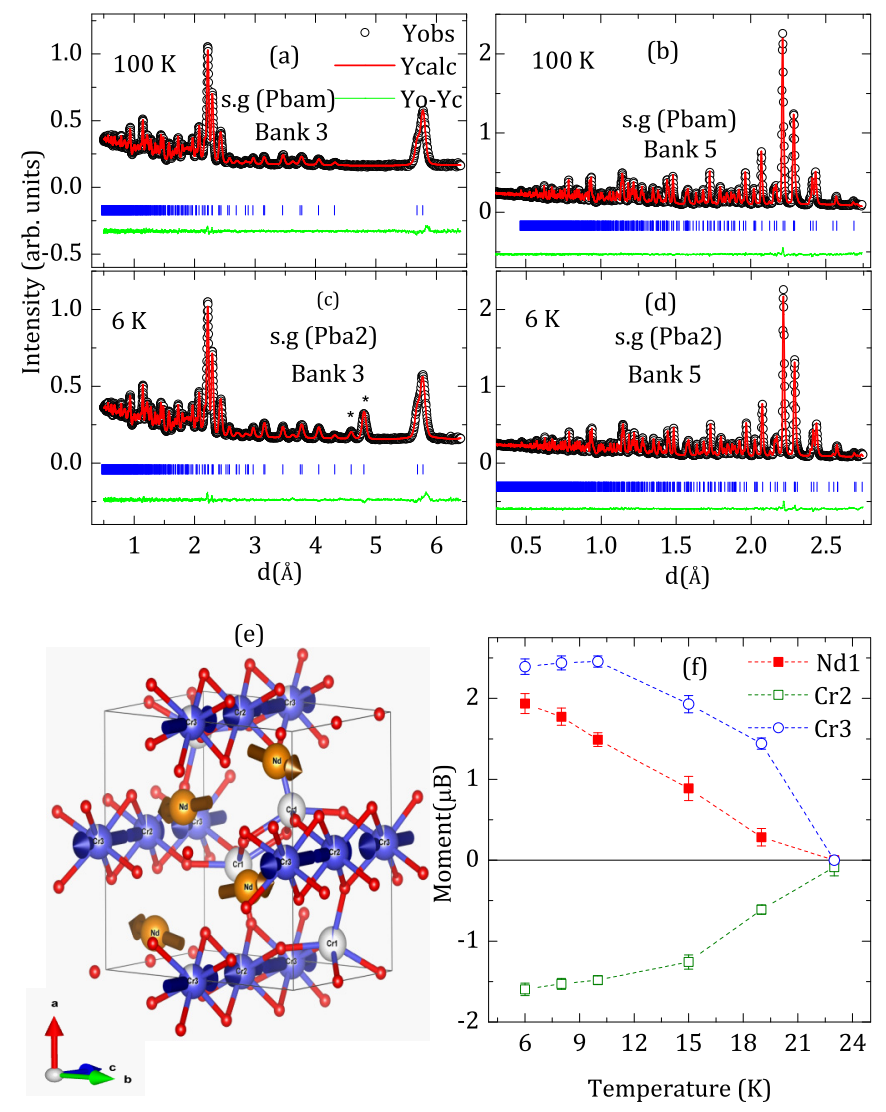

FIG. 9. (a) and (b) Rietveld refinements of the neutron diffraction data of $\mathrm{NdCrTiO}_{5}$ at $100 \mathrm{~K}$ using Pbam. (c) and (d) Rietveld refinements of the neutron diffraction pattern of $\mathrm{NdCrTiO}_{5}$ at $6 \mathrm{~K}$ using the $P b a 2$ space group. (e) Magnetic structure of $\mathrm{NdCrTiO}_{5}$ in the Pba2 space group. (f) Magnetic moment as a function of temperature at $\mathrm{Nd}$ and $\mathrm{Cr}$ sites in $\mathrm{NdCrTiO}_{5}$. 
TABLE I. Refined structural parameters and agreement factors at 100 and $6 \mathrm{~K}$, from TOF NPD data.

\begin{tabular}{|c|c|c|c|c|c|c|c|c|c|}
\hline \multicolumn{10}{|c|}{ Space group } \\
\hline \multicolumn{5}{|c|}{$(100 \mathrm{~K})$ Pbam } & \multicolumn{5}{|c|}{ (6 K) $\mathrm{Pba} 2$} \\
\hline Atoms & $x$ & $y$ & $z$ & site & Atoms & $x$ & $y$ & $z$ & site \\
\hline $\mathrm{Nd}$ & $0.14102(10)$ & $0.17272(9)$ & 0.00000 & $4 \mathrm{~g}$ & $\mathrm{Nd}$ & $0.14132(9)$ & $0.17216(8)$ & 0.17094 & $4 \mathrm{c}$ \\
\hline $\mathrm{Ti}$ & $0.11750(2)$ & $0.85601(17)$ & 0.50000 & $4 \mathrm{~h}$ & $\mathrm{Ti}$ & $0.11708(19)$ & $0.85639(15)$ & 0.67200 & $4 \mathrm{c}$ \\
\hline $\mathrm{Cr}$ & $0.11750(2)$ & $0.85601(17)$ & 0.50000 & $4 \mathrm{~h}$ & $\mathrm{Ti}$ & 0.00000 & 0.50000 & 0.42490 & $2 b$ \\
\hline $\mathrm{Ti}$ & 0.00000 & 0.50000 & $0.2489(3)$ & $4 \mathrm{f}$ & $\mathrm{Cr}$ & $0.11708(19)$ & $0.85639(15)$ & $0.672(2)$ & $4 c$ \\
\hline $\mathrm{Cr}$ & 0.00000 & 0.50000 & $0.2489(3)$ & $4 \mathrm{f}$ & $\mathrm{Cr}$ & 0.00000 & 0.50000 & $0.4249(12)$ & $2 b$ \\
\hline $\mathrm{O}$ & $0.10313(7)$ & $0.71153(7)$ & $0.25438(15)$ & $8 \mathrm{i}$ & $\mathrm{O}$ & $0.1065(4)$ & $0.7095(4)$ & $0.4275(10)$ & $4 g$ \\
\hline $\mathrm{O}$ & $0.16264(14)$ & $0.44513(10)$ & 0.00000 & $4 \mathrm{~g}$ & $\mathrm{O}$ & $0.16330(13)$ & $0.4451(10)$ & $0.1654(11)$ & $4 \mathrm{c}$ \\
\hline $\mathrm{O}$ & $0.16053(15)$ & $0.42644(11)$ & 0.50000 & $4 \mathrm{~h}$ & $\mathrm{O}$ & $0.16074(14)$ & $0.42642(10)$ & $0.6683(12)$ & $4 c$ \\
\hline \multirow[t]{5}{*}{$\mathrm{O}$} & 0.00000 & 0.00000 & $0.28280(2)$ & $4 \mathrm{e}$ & $\mathrm{O}$ & 0.00000 & 0.00000 & $0.4517(11)$ & $2 \mathrm{a}$ \\
\hline & & & & & $\mathrm{Ti}$ & 0.00000 & 0.50000 & $0.9314(15)$ & $2 b$ \\
\hline & & & & & $\mathrm{Cr}$ & 0.00000 & 0.50000 & $0.9314(15)$ & $2 b$ \\
\hline & & & & & $\mathrm{O}$ & $0.0999(4)$ & $0.7121(4)$ & $0.9183(9)$ & $4 c$ \\
\hline & & & & & $\mathrm{O}$ & 0.00000 & 0.00000 & $0.8876(13)$ & $2 \mathrm{a}$ \\
\hline$R w p$ & 2.24 & & & & & 2.02 & & & \\
\hline $\operatorname{Rexp}$ & 1.90 & & & & & 1.36 & & & \\
\hline$\chi^{2}$ & 1.389 & & & & & 2.192 & & & \\
\hline
\end{tabular}

TOF NPD data, at $6 \mathrm{~K}$, for the two different banks. It is found that our XRD refined outcomes are consistent with the NPD results. All the reflections in the NPD pattern matches with previous NPD data on this compound [26]. Magnetic reflections appear at $6 \mathrm{~K}$ [see the peaks marked with an asterisk in Fig. 9(c)], corresponding to a propagation vector $k=\left(\begin{array}{lll}0 & 0 & 0\end{array}\right)$. Magnetic structure solutions have been carried out considering the Pba2 space group, showing a very good, Rietveld refinement for both nuclear and magnetic structures. Figure 9(e) shows the refined magnetic structure in the Pba2 space group, at $6 \mathrm{~K}$, while fitted structural parameters are shown in Table I. The magnetic moments of the $\mathrm{Cr}^{+3}$ at the $2 \mathrm{~b}$ sites are antiferromagnetically oriented to each other, along the $c$ axis. The $\mathrm{Nd}^{+3}$ magnetic moments at $4 \mathrm{c}$ sites are aligned towards the $b$ axis, with a small canting to the $a$ axis, and they present antiferromagnetic coupling between infinite zigzag chains of $\mathrm{Nd}$ atoms, which extend along the $a$ axis. The temperature dependence for the magnetic moments of the $\mathrm{Nd}$ and the $\mathrm{Cr}$ atoms is presented in Fig. 9(f). The magnetic moment at the $\mathrm{Cr} 2$ site is showing negative values, while the $\mathrm{Cr} 3$ has positive values, indicating the antiparallel alignment between both sites. Most importantly, we observe that the $\mathrm{Nd}^{+3}$ and the $\mathrm{Cr}^{+3}$ moments order simultaneously, at $T_{N}$ ( $21 \mathrm{~K})$. In $\mathrm{NdCrTiO}_{5}$, whether the $\mathrm{Cr}$ atoms order first and induce ordering in the $\mathrm{Nd}$ atoms or both order simultaneously has remained controversial $[8,9,12,27]$. At $T_{N}$, the magnetic moment at the $\mathrm{Nd}^{+3}$ site is quite small in comparison to the $\mathrm{Cr}^{+3}$ magnetic moment. Both the $\mathrm{Cr} 3$ and the $\mathrm{Nd}$ moments increase with a lowering of temperature, and at temperatures below $15 \mathrm{~K}$ (but above $10 \mathrm{~K}$ ) the $\mathrm{Cr}$ moments saturate while the $\mathrm{Nd}$ moments continue to grow, down to $6 \mathrm{~K}$. The whole scenario obtained through the TOF NPD measurements clearly indicates a noncollinear arrangement of the $\mathrm{Nd}$ and the $\mathrm{Cr}$ moments in $\mathrm{NdCrTiO}_{5}$, and it supports the claim by Kori et al. [12] regarding the involvement of both the $\mathrm{Nd}$ and $\mathrm{Cr}$ atoms in the formation of AFM magnetic ordering at the Néel temperature.

\section{DISCUSSION AND CONCLUSIONS}

In reference to the structural phase transition present in $\mathrm{NdCrTiO}_{5}$ near the magnetic ordering transition temperature, Hwang et al. [8] also discussed structural transition possibilities (not shown). The hysteresis in our magnetization data was a shred of evidence to the claim of a first-order phase transition, so we decided to perform a detailed structural analysis of $\mathrm{NdCrTiO}_{5}$. Fitting of XRD data using the centrosymmetric space group Pbam in the whole temperature range results in NTE behavior below $25 \mathrm{~K}$. Such anomalies in lattice parameters, in the lower-temperature (near $T_{N}$ ) region, in the $R \mathrm{Mn}_{2} \mathrm{O}_{5}$ (where $R$ is Tb,Ho,Dy) family [5,22], have also been observed earlier. Previously, the literature on manganites has reported such unusual lattice deformation at lower temperatures, which were found to be associated with the magnetic order [28]. In the cases of $\mathrm{La}_{0.83} \mathrm{Sr}_{0.17} \mathrm{MnO}_{3}$ and $\mathrm{Pa}_{0.7} \mathrm{Sr}_{0.3} \mathrm{MnO}_{3}$, these types of changes in lattice behavior with respect to temperature have been found to be associated with delocalization of the charge carriers and structural phase transition [29,30]. In the case of $\mathrm{TbMn}_{2} \mathrm{O}_{5}$, a structural change from the centrosymmetric space group to the noncentrosymmetric space group ( $\mathrm{Pbam}$ to $\mathrm{Pb2}{ }_{1} \mathrm{~m}$ ) at the ferroelectric transition has been reported [31]. However, very small anomalies in the lattice parameters and the volume have been observed [5]. Considering our structural, dielectric, and magnetic observations and the relevant literature [5,22], we performed a symmetry analysis and looked for the possible noncentrosymmetric subspace groups of the Pbam space group; Pba2 was found. As a consequence of the strong spin lattice coupling present at almost the same temperatures, the spectral background in Raman scattering measurements suddenly increased; such 
spectral background behavior is mainly associated with the decay of the two magnons [32,33], the AFM ordering dominated by the $\mathrm{Cr}$ moments occurs at around $21 \mathrm{~K}$, and the $\mathrm{Nd}$ moments dominate below $14 \mathrm{~K}$. This is supported also by our NPD data.

In conclusion, we have confirmed magnetic and dielectric anomalies in $\mathrm{NdCrTiO}_{5}$ below $21 \mathrm{~K}$. Structural anomalies near the AFM ordering show the same trend as in the derivative of the $M(T)$ curve, indicating a strong spin lattice coupling in $\mathrm{NdCrTiO}_{5}$. A change of space group symmetry and NTE is observed near the ferroelectric transition temperature, which is noncentrosymmetric (Pba2) in contrast to the roomtemperature centrosymmetric space group. Raman measurements also support the observation of a space group change at low temperatures and strong spin lattice coupling. Phonon modes involving the $\mathrm{Nd}$ and the $\mathrm{Cr}$ atoms are particularly sensitive to the magnetic ordering. Neutron diffraction data confirm the simultaneous magnetic ordering of the $\mathrm{Nd}$ and the $\mathrm{Cr}$ atoms at Néel temperature and a noncollinear arrangement at $6 \mathrm{~K}$. Below $15 \mathrm{~K}$, the $\mathrm{Nd}$ and the $\mathrm{Cr}$ orderings do not follow the same temperature dependence, thereby indicating the presence of a complex $f-d$ exchange interaction. This supports the idea that the spontaneous electric polarization in a magnetically ordered state in this compound is invoked by the magnetic ordering in a noncentrosymmetric space group, which points toward DM interaction being responsible for the multiferroicity in this compound.

\section{ACKNOWLEDGMENTS}

The authors are thankful to Rajeev Rawat for providing the superconducting cryomagnet used for magnetodielectric measurements, J. A. Q. Coaquira for providing magnetization measurements, G. Sharma and S. Tyagi for help during Raman measurements, and Rajamani Raghunathan for fruitful discussions. K.G. acknowledges the Council of Scientific and Industrial Research, New Delhi, India for support in the form of SRF. K.G. and D.K.S. gratefully acknowledge support from INSPIRE faculty award (IFA-13/PH-52), and DST-DESY project for financial support for performing synchrotron $\mathrm{X}$-ray diffraction studies. Part of this research was carried out at the PETRA III synchrotron radiation source at DESY, a member of Helmholtz Association. The work at the ISIS Neutron and Muon facility was supported by the science and technology facilities council and DST-RAL project.
[1] T. Kimura, T. Goto, H. Shintani, K. Ishizaka, T.-H. Arima, and Y. Tokura, Nature (London) 426, 55 (2003).

[2] W. Eerenstein, N. Mathur, and J. F. Scott, Nature (London) 442, 759 (2006).

[3] N. A. Spaldin, S.-W. Cheong, and R. Ramesh, Phys. Today 63(10), 38 (2010).

[4] M. Fukunaga, Y. Sakamoto, H. Kimura, Y. Noda, N. Abe, K. Taniguchi, T. Arima, S. Wakimoto, M. Takeda, K. Kakurai et al., Phys. Rev. Lett. 103, 077204 (2009).

[5] G. R. Blake, L. C. Chapon, P. G. Radaelli, S. Park, N. Hur, S. W. Cheong, and J. Rodriguez-Carvajal, Phys. Rev. B 71, 214402 (2005).

[6] N. Hur, Nature (London) 429, 392 (2004).

[7] C. Wang, G.-C. Guo, and L. He, Phys. Rev. Lett. 99, 177202 (2007).

[8] J. Hwang, E. S. Choi, H. D. Zhou, J. Lu, and P. Schlottmann, Phys. Rev. B 85, 024415 (2012).

[9] J. Saha, G. Sharma, and S. Patnaik, J. Magn. Magn. Mater. 360, 34 (2014).

[10] T. Moriya, Phys. Rev. 120, 91 (1960).

[11] J. B. Goodenough, Phys. Rev. 100, 564 (1955).

[12] S. Kori, T. Okamura, R. Okazaki, I. Terasaki, and Y. Yasui, Phys. Rev. B 91, 144403 (2015).

[13] A.-C. Dippel, H.-P. Liermann, J. T. Delitz, P. Walter, H. SchulteSchrepping, O. H. Seeck, and H. Franz, J. Synch. Radiat. 22, 675 (2015).

[14] A. C. Hannon, Nucl. Instrum. Methods Phys. Res. A 551, 88 (2005).

[15] R. Ranjan and D. Pandey, J. Phys.: Condens. Matter 13, 4239 (2001).

[16] H. Rietveld, Acta Crystallogr. 22, 151 (1967).

[17] R. E. Marsh, Acta Crystallogr. Sect. B 42, 193 (1986).

[18] R. E. Marsh, Acta Crystallogr. Sect. B 51, 897 (1995).
[19] A. Indra, K. Dey, S. Majumdar, I. Sarkar, S. Francoual, R. P Giri, N. Khan, P. Mandal, and S. Giri, Phys. Rev. B 95, 094402 (2017).

[20] A. Indra, K. Dey, J. Dey, S. Majumdar, U. Rütt, O. Gutowski, M. v. Zimmermann, and S. Giri, Phys. Rev. B 98, 014408 (2018).

[21] Bilbao Crystallographic Server, http://www.cryst.ehu.es (2019).

[22] L. C. Chapon, G. R. Blake, M. J. Gutmann, S. Park, N. Hur, P. G. Radaelli, and S.-W. Cheong, Phys. Rev. Lett. 93, 177402 (2004)

[23] A. Litvinchuk, J. Magn. Magn. Mater. 321, 2373 (2009).

[24] T. Basu, K. Singh, S. Gohil, S. Ghosh, and E. Sampathkumaran, J. Appl. Phys. 118, 234103 (2015).

[25] T. Basu, D. T. Adroja, F. Kolb, H.-A. Krug von Nidda, A. Ruff, M. Hemmida, A. D. Hillier, M. Telling, E. V. Sampathkumaran, A. Loidl, and S. Krohns, Phys. Rev. B 96, 184431 (2017).

[26] G. Buisson, J. Phys. Chem. Solids 31, 1171 (1970).

[27] X. Qian, Y. Fang, J. Kang, S. Cao, and J. Zhang, Physica B 495, 1 (2016).

[28] A. Arulraj, A. Biswas, A. K. Raychaudhuri, C. N. R. Rao, P. M. Woodward, T. Vogt, D. E. Cox, and A. K. Cheetham, Phys. Rev. B 57, R8115(R) (1998).

[29] B. Garcia-Landa, M. R. Ibarra, J. D. De Teresa, G.-m. Zhao, K. Conder, and H. Keller, Solid State Commun. 105, 567 (1998).

[30] A. Asamitsu, Y. Moritomo, R. Kumai, Y. Tomioka, and Y. Tokura, Phys. Rev. B 54, 1716 (1996).

[31] C. Wang, G.-C. Guo, and L. He, Phys. Rev. B 77, 134113 (2008).

[32] S. Gohil, K. K. Iyer, P. Aswathi, S. Ghosh, and E. Sampathkumaran, J. Appl. Phys. 108, 103517 (2010).

[33] M. Lacerda, F. Kargar, E. Aytan, R. Samnakay, B. Debnath, J. Li, A. Khitun, R. Lake, J. Shi, and A. Balandin, Appl. Phys. Lett. 110, 202406 (2017). 